\title{
Prognostic value of serum lactate dehydrogenase in hospitalized patients with Covid-19
}

\author{
Vicente Aguadero*, Elisa Nuez, Carlos García-Miralles, Gemma Sole-Enrech, \\ Ruth Cano-Corres
}

Clinical Laboratory. Parc Tauli Hospital Universitari. Institut d'Investigació i Innovació Parc Taulí

I3PT. Universitat Autònoma de Barcelona. Sabadell, Spain

\begin{abstract}
Background: Biochemical markers in COVID-19 remain to be defined. We analyzed the usefulness of LDH and ferritin in predicting outcome. Methods: This retrospective study analyzed ferritin and LDH concentrations obtained during the first 11 days of hospitalization in COVID-19 patients. We compared the change in ferritin and LDH concentrations obtained on each day of hospital admission with respect to baseline values between patients with favorable and unfavorable outcomes. We used receiver operating curve analysis to determine cutoffs for predicting outcomes. Results: We analyzed 387 patients. For determinations done on the 9th day, increases in LDH concentrations $>14.6 \%$ over the baseline yielded $80 \%$ positive predictive value, and a lack of increase yielded $96 \%$ negative predictive value for unfavorable outcomes. The change in ferritin concentration yielded lower predictive values. Conclusion: The percentage of change in $\mathrm{LDH}$ with respect to the baseline on the 9 th day of hospitalization can predict outcome..
\end{abstract}

Keywords: LDH, COVID-19, prognosis, hospitalization, ferritin

Received: $31^{\text {st }}$ December 2020; Accepted: 20 th March 2021; Published: $27^{\text {th }}$ March 2021

\section{Introduction}

Since the first detected infections with the SARS-CoV-2 virus in China in December 2019, the COVID-19 outbreak has developed into a pandemia (1-7). The high infectivity of the virus, lack of effective treatment or vaccines, and the potential for spread by asymptomatic carriers have made the management of COVID-19 extremely challenging $(1,2,6,8,9)$.

Spain is one of the European countries where COVID-19 has had the greatest impact, main- ly due to early spreading when the disease was still poorly understood. At the time of writing (2020/07/14), 256.619 diagnosed cases and over 28,400 deaths have been confirmed in Spain (10). Within Spain, the Metropolitan Area of Barcelona has been one of the hardest hit areas, with 38,442 confirmed cases in a population of 3.2 million people (11).

The clinical course of COVID-19 ranges from asymptomatic infection or mild respiratory symptoms to interstitial pneumonia resulting in

\footnotetext{
* Corresponding author: Vicente Aguadero, Clinical Laboratory, Parc Taulí Hospital Universitari, Parc Tauli, 1, 08208, Sabadell (Barcelona), Spain. E-mail: vaguadero@tauli.cat
} 
respiratory failure with intravascular coagulopathies, sometimes leading to multiple organ failure and death $(1-6,8,9)$. The main reason for hospitalization is acute respiratory failure (1), and complications often require long stays and admission to intensive care units (ICU) $(6,8,9)$. Patients with severe COVID-19 often have elevated serum biomarkers of inflammation [ferritin $(2,3,6-9,12,13)$ and C-reactive protein $(2-5,8$, $9,12-14)$ ], cardiac and muscle injury [troponin $(3,5-9,12)$, creatinine kinase $(3,8,9,12)$, and lactate dehydrogenase $(\mathrm{LDH})(3,5,6,8,9,12$, $13)]$, liver and kidney function [creatinine $(3,6$, $8,9,12)$ and transaminases $(3,5,8,12,13)]$, and hypoxia [lactate (14)]. In our hospital, the most characteristic laboratory findings in patients with severe COVID-19 have been significant increases in the acute-phase reactant ferritin and in the muscle injury marker LDH.

Scant information is available about the usefulness of biochemical markers in predicting the development of severe and critical COVID-19 $(3,4,6-8,12)$. We aimed to analyze the utility of ferritin and LDH in predicting the course of COVID-19 and in identifying patients at higher risk of adverse outcomes.

\section{Methods}

\section{Study design and ethics committee approval}

This retrospective study analyzed laboratory and clinical data from COVID-19 patients obtained from the laboratory informatics system (SmartLis ${ }^{\circledR}$, Lab Technologies SA, Lugano, Switzerland) and the hospital's electronic medical record system. Our hospital's ethics committee approved the study.

\section{Patients}

We included all patients admitted to any ward between March 15, 2020 and April 15, 2020 who met the following criteria: a) PCR positive for SARS-CoV-2, b) fever and/or respiratory failure and/or radiologically confirmed pneumonia, and c) at least one determination of ferritin or $\mathrm{LDH}$ within the first 72 hours of hospitalization (baseline values).

\section{Variables}

We recorded the following data for each patient: age, sex, date of hospitalization, and serum ferritin and/or LDH concentrations obtained within the first 11 days of hospitalization. We calculated the percentage of change (increase or decrease) between baseline measurements of ferritin and LDH (obtained in the first 72 hours) and subsequent determinations. $\mathrm{LDH}$ and ferritin were analyzed in serum obtained in tubes with separating gel without additives (VACUETTE®, Greiner Bio-One International GmbH, Kremsmünster, Austria) using Cobas ${ }^{\circledR} 8000$ c701 or Cobas 6000 c502 analyzers (Roche Diagnostics, Mannheim, Germany). The results obtained with the two analyzers are interchangeable.

We classified outcomes as favorable when patients were discharged from the hospital without requiring ICU admission and as unfavorable when patients required admission to the ICU or died. In patients whose outcomes were classified as unfavorable, we analyzed only LDH and ferritin determinations obtained before any criteria for unfavorable outcome were met.

\section{Statistical analysis}

To compare frequencies between patients with favorable versus unfavorable outcomes and between males and females, we used chi-square tests. To compare mean age between patients with favorable outcomes versus those with unfavorable outcomes, we used Student's t-test.

To compare mean baseline concentrations and percentage variation in LDH and ferritin on each day of hospitalization between patients with favorable and unfavorable outcomes, we used Student's t-tests or Mann-Whitney U tests as appropriate according to the results of the Kolm- 
ogorov-Smirnov test. Statistical significance was set at $\mathrm{p}<0.05$. When the mean concentrations or percentage variations of $\mathrm{LDH}$ or ferritin differed significantly between patients with favorable and unfavorable outcomes, we elaborated receiver operating characteristic (ROC) curves with unfavorable outcome as the state variable. To determine prognostic cutoffs with good specificity, moderate sensitivity, and optimal positive predictive value (PPV), we calculated the area under the curve (AUC).

To perform the multiple pairs comparison of percentage variation means of LDH and Ferritin from baseline values between the different days of hospitalization for favorable and unfavorable outcome, we used Bonferroni test. Statistical significance was set at $\mathrm{p}<0.05$.

We used SPSS Statistics version 25.0 (IBM; Armonk, NY, USA) for all analyses.

\section{Results}

\section{Patients and baseline concentrations}

A total of 386 patients [median age, 66 y; 236 (61\%) men and $150(39 \%)$ women] met the inclusion criteria; 314 (81\%) had favorable outcomes and 72 (19\%) had unfavorable outcomes (Table 1). Of those with unfavorable outcomes, $15(21 \%)$ patients were discharged to the wards after ICU admission and 58 (79\%) patients died. The global mortality rate within the study population was $15 \%$.

Compared to patients with favorable outcomes, patients with unfavorable outcomes were older (mean age, 69 vs. 65 years, $\mathrm{p}=0.033$ ). The proportions of men and women with unfavorable outcomes were not significantly different $(22 \%$ vs. $14 \%$, respectively, $\mathrm{p}=0.127$ ) (Table 1 ).

We analyzed a total of $358 \mathrm{LDH}$ determinations, 278 from patients with favorable outcomes and 80 from patients with unfavorable outcomes, and 543 ferritin determinations, 428 from patients with favorable outcomes and 115 from patients with unfavorable outcomes. Due to the low number of LDH and ferritin determinations in patients with unfavorable outcome during the first days of hospitalization, we combined the data from the first three days. Likewise, because only one LDH determination and three ferritin determinations were recorded on the $8^{\text {th }}$ day in patients with an unfavorable outcome, we combined the data from the $7^{\text {th }}$ and $8^{\text {th }}$ days.

Patients with unfavorable outcomes had higher baseline concentrations of LDH $[437 \mathrm{U} / \mathrm{L}$ vs. $311 \mathrm{U} / \mathrm{L}$ in those with favorable outcomes, $\mathrm{p}<0.001]$ and ferritin [1652 $\mathrm{ng} / \mathrm{mL}$ vs. 1244 $\mathrm{ng} / \mathrm{mL}$ in patients with favorable outcomes, $\mathrm{p}=0.009$ ] (Table 2).

\section{Percentage of change from baseline values}

Table 3 reports the mean percentage change with respect to baseline values of $\mathrm{LDH}$ and ferritin for determinations obtained on different days of hospitalization, comparing this variable between patients with favorable outcomes and those with unfavorable outcomes.

Table 1. Demographic characteristics of hospitalized Covid-19 patients admitted to the study. FO: Favorable outcome. UO: Unfavorable outcome. y: years. IQR: interquartile range.

\begin{tabular}{|c|c|c|c|c|c|}
\hline & \multirow{2}{*}{$\begin{array}{c}\text { AGE mean } \\
\text { y (IQR) }\end{array}$} & \multicolumn{4}{|c|}{ SEX No. (\%) } \\
\hline & & P Value & Men & Female & P Value \\
\hline TOTAL $(n=386)$ & $66(52-80)$ & & $236(61 \%)$ & $150(39 \%)$ & \\
\hline $\mathrm{FO}(\mathrm{n}=314)$ & $65(43-87)$ & \multirow{2}{*}{0.033} & $185(78 \%)$ & $129(86 \%)$ & \multirow{2}{*}{0.127} \\
\hline $\mathrm{UO}(\mathrm{n}=72)$ & $69(50-88)$ & & $51(22 \%)$ & $21(14 \%)$ & \\
\hline
\end{tabular}

$\mathrm{P}$ values indicate differences between FO and UO patients. $\mathrm{P}<.05$ was considered statistically significant. 
Table 2. Baseline ( $\leq 72 \mathrm{~h}$ of admission) concentrations of $\mathrm{LDH}$ and ferritin.

\begin{tabular}{|c|c|c|c|c|c|c|c|c|c|c|c|}
\hline \multirow{2}{*}{ TEST } & \multicolumn{2}{|r|}{ FO } & \multicolumn{2}{|r|}{ UO } & \multirow{2}{*}{$\begin{array}{c}P- \\
\text { value }\end{array}$} & \multirow{2}{*}{$\operatorname{AUC}(95 \% \mathrm{CI})$} & \multirow{2}{*}{$\begin{array}{l}\text { CUT- } \\
\text { OFF }\end{array}$} & \multirow{2}{*}{$\begin{array}{c}S \\
(\%)\end{array}$} & \multirow{2}{*}{$\begin{array}{l}\text { Sp } \\
(\%)\end{array}$} & \multirow{2}{*}{$\begin{array}{l}\text { PPV } \\
(\%)\end{array}$} & \multirow{2}{*}{$\begin{array}{l}\text { NPV } \\
(\%)\end{array}$} \\
\hline & n & Mean & n & Mean & & & & & & & \\
\hline \multirow{3}{*}{ LDH } & \multirow{3}{*}{243} & \multirow{3}{*}{$311 \mathrm{u} / \mathrm{L}$} & \multirow{3}{*}{64} & \multirow{3}{*}{$437 \mathrm{u} / \mathrm{L}$} & \multirow{3}{*}{0.001} & \multirow{3}{*}{$0.705(0.625-0.784)$} & 300 & 73 & 55 & 30 & 89 \\
\hline & & & & & & & 410 & 50 & 87 & 50 & 87 \\
\hline & & & & & & & 510 & 31 & 92 & 55 & 83 \\
\hline \multirow{2}{*}{ Ferritin } & \multirow{2}{*}{307} & \multirow{2}{*}{$1244 \mathrm{ng} / \mathrm{mL}$} & \multirow{2}{*}{80} & \multirow{2}{*}{$1652 \mathrm{ng} / \mathrm{mL}$} & \multirow{2}{*}{0.009} & \multirow{2}{*}{$0.594(0.522-0.666)$} & 1300 & 50 & 62 & 25 & 82 \\
\hline & & & & & & & 2073 & 30 & 84 & 33 & 82 \\
\hline
\end{tabular}

FO: Favorable outcome. UO: Unfavorable outcome. AUC: area under the receiver operating characteristic curve. CI: confidence interval. S: sensitivity. Sp: specificity. PPV: positive predictive value. NPV: negative predictive value. P values indicate differences between $\mathrm{FO}$ and $\mathrm{UO}$ patients. $\mathrm{P}<.05$ was considered statistically significant.

Table 3. Mean differences in LDH and ferritin concentrations with respect to baseline values by days of hospitalization for patients with favorable and unfavorable outcomes.

\begin{tabular}{|c|c|c|c|c|c|c|c|c|c|c|}
\hline \multirow{3}{*}{ Day of hospitalization } & \multicolumn{4}{|c|}{ FERRITIN } & \multirow[b]{3}{*}{ P-value } & \multicolumn{4}{|c|}{ LDH } & \multirow[b]{3}{*}{ P-value } \\
\hline & \multicolumn{2}{|c|}{ FO } & \multicolumn{2}{|c|}{ UO } & & \multicolumn{2}{|c|}{ FO } & \multicolumn{2}{|c|}{ UO } & \\
\hline & $\mathbf{N}$ & $\begin{array}{c}\text { Mean } \\
(\%)\end{array}$ & $\mathbf{N}$ & $\begin{array}{c}\text { Mean } \\
(\%)\end{array}$ & & $\mathbf{N}$ & $\begin{array}{c}\text { Mean } \\
(\%)\end{array}$ & $\mathbf{N}$ & $\begin{array}{c}\begin{array}{c}\text { Mean } \\
(\%)\end{array} \\
\end{array}$ & \\
\hline Between 1st and 3rd & 28 & 6.7 & 20 & 12.6 & $0.517^{*}$ & 20 & 0.23 & 12 & -9.82 & $0.632^{\#}$ \\
\hline 4 th & 46 & 5.9 & 14 & -11 & $0.081^{\#}$ & 27 & 3.5 & 11 & 1.41 & $0.899^{\#}$ \\
\hline $5 \mathrm{st}$ & 51 & 12.2 & 20 & 26 & $0.335^{*}$ & 30 & 4.5 & 16 & 11.6 & $0.333^{\#}$ \\
\hline 6th & 51 & -3.8 & 12 & 51.9 & $0.1^{\#}$ & 32 & -17.6 & 6 & 20.5 & $0.232^{*}$ \\
\hline Between 7th and 8th & 124 & 16.33 & 18 & 45 & $0.067^{\#}$ & 85 & -2.7 & 11 & 19.2 & $0.047^{\#}$ \\
\hline 9th & 41 & 36.7 & 14 & 60 & $0.008^{\#}$ & 25 & -16.5 & 10 & 56.8 & $0.001^{\#}$ \\
\hline 10th & 55 & 44 & 10 & 148 & $0.136^{\#}$ & 41 & -3.5 & 7 & 50 & $0.108^{*}$ \\
\hline 11th & 32 & 50 & 7 & 36 & $0.507^{\#}$ & 18 & -8.9 & 7 & 24.7 & $0.467^{\#}$ \\
\hline & 428 & & 115 & & & 278 & & 80 & & \\
\hline
\end{tabular}

Mean: mean percentage change with respect to the baseline values. FO: Favorable outcome. UO: Unfavorable outcome. P values indicate differences between FO and UO patients. $\mathrm{P}<.05$ was considered statistically significant (are shown in bold).

*: Assessed by Student's t-test. ": Assessed by Mann-Whitney U test.

Table 4 reports the results of the ROC analysis for the days with significant differences in mean $\mathrm{LDH}$ or ferritin concentrations between the groups of patients with favorable and unfavorable outcomes.

For LDH, significant differences between the groups of patients with favorable and unfavorable outcomes were observed only on the com- bined 7th and 8th day $(\mathrm{p}=0.047)$ and on the 9th day $(\mathrm{p}=0.001)$.

Figure 1 shows the boxplots and ROC curves obtained for the percentage change in $\mathrm{LDH}$ with respect to baseline at these timepoints as unfavorable outcome marker. As we can observe in table 4 , the AUC in the ROC curve for the 9th day was 0.920 . At the cutoff 14.6 (\% of increase in LDH 
Table 3. Mean differences in LDH and ferritin concentrations with respect to baseline values by days of hospitalization for patients with favorable and unfavorable outcomes.

\begin{tabular}{|c|c|c|c|c|c|c|c|c|c|c|}
\hline \multirow{3}{*}{ Day of hospitalization } & \multicolumn{4}{|c|}{ FERRITIN } & \multirow[b]{3}{*}{ P-value } & \multicolumn{4}{|c|}{ LDH } & \multirow[b]{3}{*}{ P-value } \\
\hline & \multicolumn{2}{|c|}{ FO } & \multicolumn{2}{|c|}{ UO } & & \multicolumn{2}{|c|}{ FO } & \multicolumn{2}{|r|}{ UO } & \\
\hline & $\mathbf{N}$ & $\begin{array}{c}\text { Mean } \\
(\%)\end{array}$ & $\mathbf{N}$ & $\begin{array}{c}\text { Mean } \\
(\%)\end{array}$ & & $\mathbf{N}$ & $\begin{array}{c}\text { Mean } \\
(\%)\end{array}$ & $\mathbf{N}$ & Mean (\%) & \\
\hline Between 1st and 3rd & 28 & 6.7 & 20 & 12.6 & $0.517^{*}$ & 20 & 0.23 & 12 & -9.82 & $0.632^{\#}$ \\
\hline 4 th & 46 & 5.9 & 14 & -11 & $0.081^{\#}$ & 27 & 3.5 & 11 & 1.41 & $0.899^{\#}$ \\
\hline $5 \mathrm{st}$ & 51 & 12.2 & 20 & 26 & $0.335^{*}$ & 30 & 4.5 & 16 & 11.6 & $0.333^{\#}$ \\
\hline 6 th & 51 & -3.8 & 12 & 51.9 & $0.1^{\#}$ & 32 & -17.6 & 6 & 20.5 & $0.232^{*}$ \\
\hline Between 7th and 8th & 124 & 16.33 & 18 & 45 & $0.067^{\#}$ & 85 & -2.7 & 11 & 19.2 & $0.047^{\#}$ \\
\hline 9th & 41 & 36.7 & 14 & 60 & $0.008^{\#}$ & 25 & -16.5 & 10 & 56.8 & $0.001^{\#}$ \\
\hline 10th & 55 & 44 & 10 & 148 & $0.136^{\#}$ & 41 & -3.5 & 7 & 50 & $0.108^{*}$ \\
\hline 11th & 32 & 50 & 7 & 36 & $0.507^{\#}$ & 18 & -8.9 & 7 & 24.7 & $0.467^{\#}$ \\
\hline & 428 & & 115 & & & 278 & & 80 & & \\
\hline
\end{tabular}

Mean: mean percentage change with respect to the baseline values. FO: Favorable outcome. UO: Unfavorable outcome. P values indicate differences between FO and UO patients. $\mathrm{P}<.05$ was considered statistically significant (are shown in bold).

*: Assessed by Student's t-test. \#: Assessed by Mann-Whitney U test.

Table 4. Results of the receiver operating characteristic (ROC) analysis of the percentage of change in ferritin and LDH concentrations with respect to baseline values for the timepoints where significant differences were observed by the groups of patients with favorable and unfavorable outcomes.

\begin{tabular}{|c|c|c|c|c|c|c|c|}
\hline & $\begin{array}{c}\text { Day of } \\
\text { hospitalization }\end{array}$ & $\operatorname{AUC}(95 \% \mathrm{CI})$ & $\begin{array}{c}\text { CUTOFF } \\
(\%) \\
\end{array}$ & S (\%) & Sp (\%) & PPV (\%) & NPV (\%) \\
\hline \multirow{3}{*}{ FERRITIN } & \multirow{3}{*}{$9^{\text {th }}$} & \multirow{3}{*}{$\begin{array}{c}0.740 \\
(0.592-0.884)\end{array}$} & 9.2 & 64 & 78 & 50 & 86 \\
\hline & & & 17.7 & 57 & 83 & 53 & 85 \\
\hline & & & 63.7 & 43 & 85 & 50 & 81 \\
\hline \multirow{6}{*}{$\mathrm{LDH}$} & \multirow{3}{*}{$\begin{array}{l}\text { Between } 7^{\text {th }} \\
\text { and } 8^{\text {th }}\end{array}$} & \multirow{3}{*}{$\begin{array}{c}0.684 \\
(0.494-0.875)\end{array}$} & 4 & 64 & 69 & 22 & 92 \\
\hline & & & 19.7 & 55 & 88 & 36 & 93 \\
\hline & & & 49.3 & 27 & 94 & 38 & 90 \\
\hline & \multirow{3}{*}{$9^{\text {th }}$} & \multirow{3}{*}{$\begin{array}{c}0.920 \\
(0.835-1.000)\end{array}$} & 0.1 & 90 & 88 & 75 & 96 \\
\hline & & & 14.6 & 80 & 92 & 80 & 92 \\
\hline & & & 23.2 & 46 & 92 & 71 & 79 \\
\hline
\end{tabular}

AUC: area under the ROC curve. CI: confidence interval. S: sensitivity. Sp: specificity. PPV: positive predictive value. NPV: negative predictive value. Significant results are shown in bold. 
a)
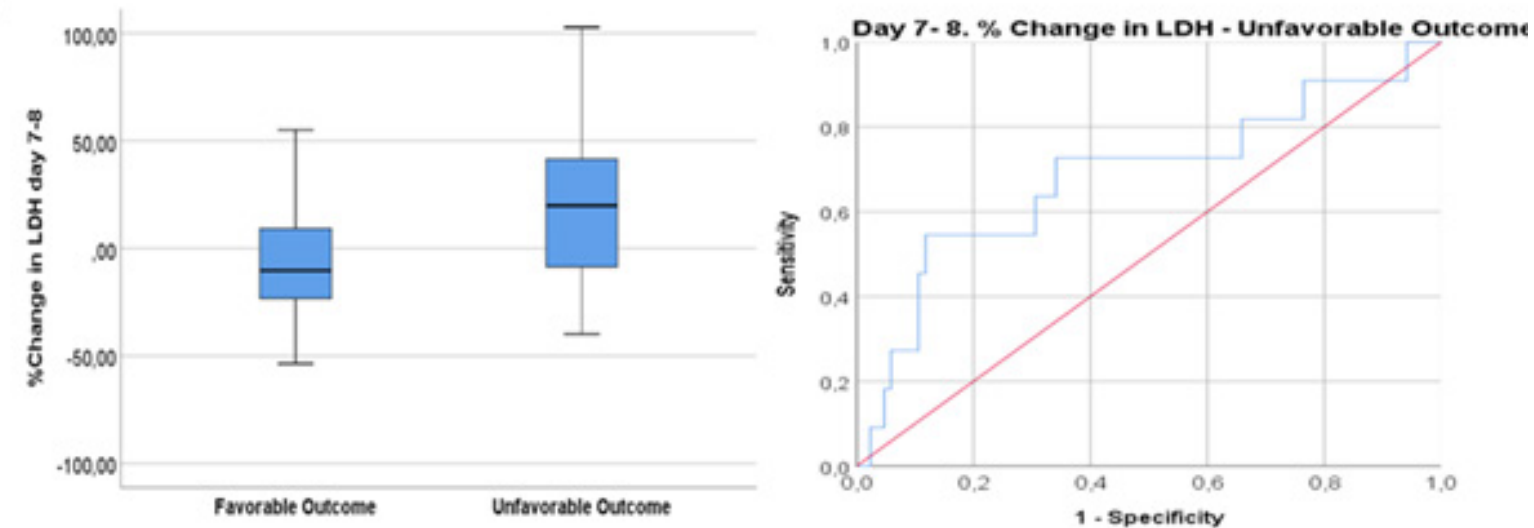

b)
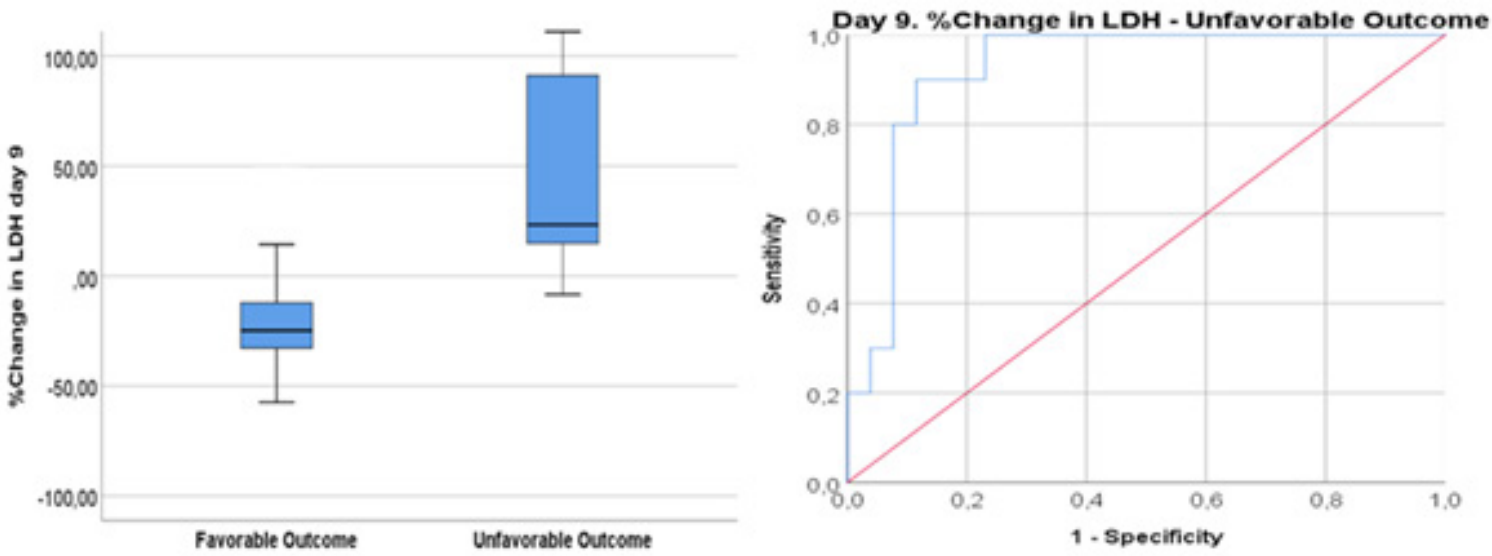

Fig.1. Boxplots and ROC curves for the percentage of change with respect to baseline values for LDH determinations obtained on days 7 or 8 of hospitalization (a) and on day 9 of hospitalization (b).

with respect to the baseline value), diagnostic accuracy was: $80 \%$ sensitivity, $92 \%$ specificity, $80 \%$ PPV and $92 \%$ negative predictive value (NPV) for unfavorable outcomes. There were only 10 patients whose LDH determinations on day 9 were above this cutoff, among them, only 2 did not develop unfavorable outcomes; the other 8 true-positive cases, all died.

On the other hand, we also observed that any increase in LDH (cutoff $<0.1 \%$ ) with respect to the baseline value at day 9 yielded $90 \%$ sensitivity, $88 \%$ specificity, $75 \% \mathrm{PPV}$, and $96 \% \mathrm{NPV}$, as unfavorable outcome marker. Among the 24 cases whose LDH determinations on day 9 were below this cutoff, only 1 had an unfavorable outcome. Thus, on day 9 of hospitalization, the absence of an increase in LDH concentration with respect to the baseline value allows fatal outcomes to be ruled out with a very low probability of error.

When comparing multiple pairs, only very close differences to significance were obtained for percentage variation means of LDH on day 9 of hospitalization with respect to the first days of hospitalization in patients with unfavorable outcome (between $1^{\text {st }}$ and $3^{\text {rd }}$ day of hospitalization vs. $9^{\text {th }}$ day of hospitalization, $p=0.063$ ). Significance differences were not obtained for patients with favorable outcome between different days of hospitalization. 
For ferritin, significant differences between the groups of patients with favorable and unfavorable outcome were found only on day 9 of hospitalization ( $\mathrm{p}=0.008)$, but the AUC in the ROC analysis was low (Figure 2). When comparing ferritin multiple pairs, significance differences between any of the hospitalization days were not obtained in both groups of patients, favorable and unfavorable outcome.

\section{Discussion}

In our research, we corroborated those of other studies about COVID-19 that identify age as a risk factor both for infection and developing complications $(6-9,13,15-22)$.

The mortality rate in our study $(15 \%)$ was slightly lower than those reported in other series of hospitalized patients $(20 \%-30 \%)(6,23,24)$.

Early, appropriate management of COVID-19 reduces mortality $(15,16)$. The main objective of our study was to find biochemical markers that could be used to predict the course of COVID-19. We evaluated serum LDH and ferritin for two reasons: first, in our experience, it seemed these parameters remained elevated for a

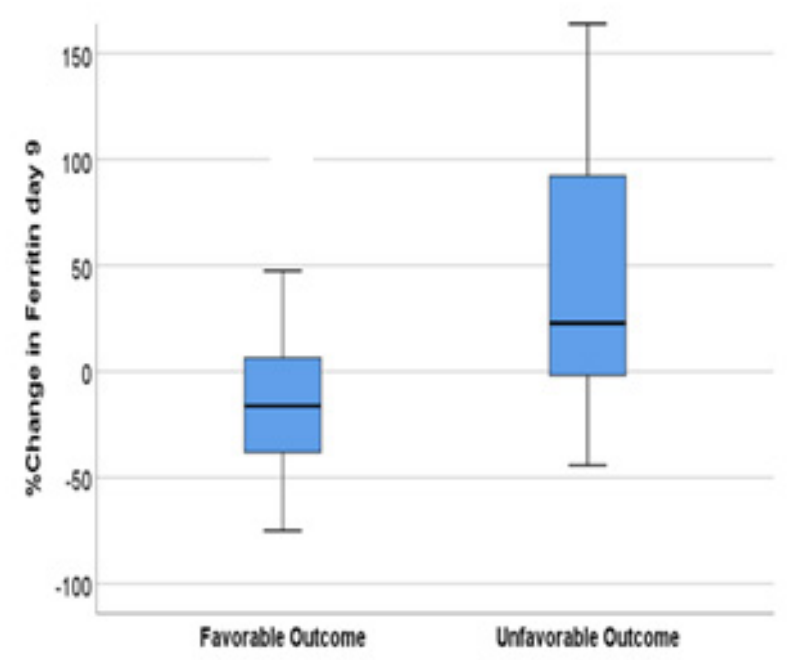

long period in hospitalized COVID-19 patients, and, second, several studies reported values of LDH and ferritin above the upper reference limit in severe cases of COVID-19 or reported significant differences in these parameters between patients who died in the hospital and those who survived (3-5, 7-9, 13, 17-19).

Although several studies reported significant differences in LDH and ferritin concentrations between severe and non-severe cases of COVID-19 at hospital admission $(3,7,9,25)$, these studies did not analyze the prognostic value of these parameters or report predictive values. We also found significant differences in baseline LDH and ferritin values at hospital admission between patients with favorable and unfavorable outcomes, but the baseline values yielded very low AUCs in the ROC analysis and did not enable us to predict prognosis. Although baseline LDH > $300 \mathrm{u} / \mathrm{L}$ yielded an NPV of $89 \%$, this parameter is unacceptable to rule out unfavorable outcomes because applying this cutoff resulted in 17 false negatives.

Most studies that evaluate the prognostic capacity of serum markers in COVID-19 consider absolute values of the parameters obtained on suc-

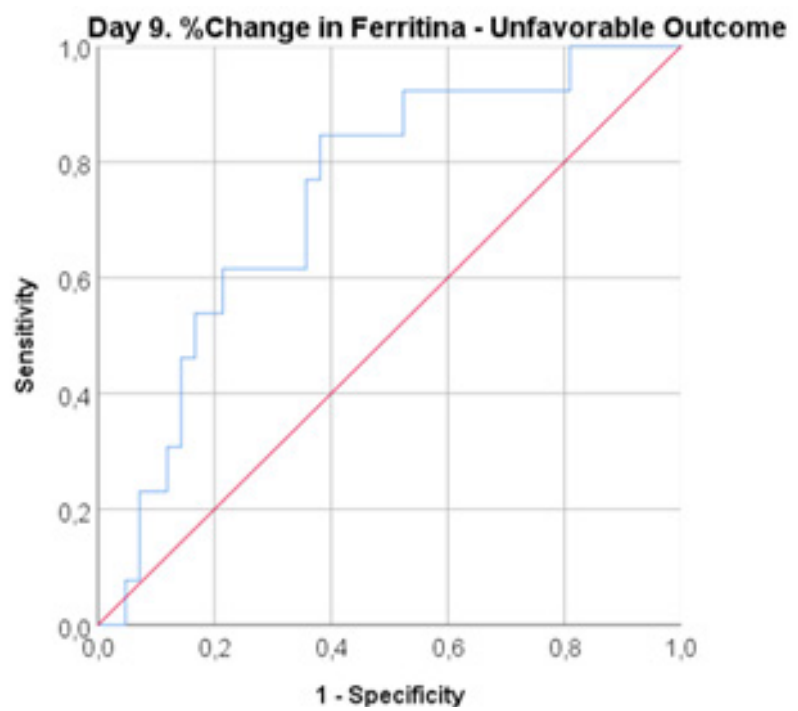

Fig. 2. The box plot and the ROC curve for Ferritin data obtained at $9^{\text {th }}$ day of hospitalization. 
cessive days of hospitalization. This approach ignores baseline values of these parameters and fails to take patients' initial clinical situation into account (17-20, 26-28). By contrast, the present study used the percentage of variation with respect to the baseline value. We believe that this approach increases the sensitivity of the markers and can help predict unfavorable outcomes earlier.

During the first 6 days of hospitalization, no significant differences in percentage variations of LDH or ferritin were found between patients with favorable outcomes and those with unfavorable outcomes. These findings are likely due to two characteristics of severe COVID-19. First, patients with severe COVID-19 often require long hospital stays (survivors averaging 17 days in hospital and 14 days in the ICU (25), fundamentally due to the lack of a really effective treatment) $(29,30)$. And second, many patients' condition worsens significantly after the second week of hospitalization (30-32). We observed that after the $6^{\text {th }}$ day of hospitalization the percentage change in LDH started to diverge remarkably between patients with favorable outcomes and those with unfavorable outcomes. The difference was negative in patients with favorable outcomes but positive in those with unfavorable outcomes (Table 3), and these differences became significant from the $7^{\text {th }}$ day of hospitalization.

Wang et al. (20) and Huang et al. (22) pointed to the good prognostic capacity of $\mathrm{LDH}$, reporting that patients who were admitted to the ICU or patients with severe forms of disease had higher LDH mean values (2.1-fold and 1.4-fold, respectively).

All 8 true-positive cases identified with the $14.6 \%$ cutoff for percentage change in LDH died. Thus, LDH was not only a good prognostic marker for unfavorable outcome, but it may also be a marker for mortality. Although the design of our study cannot prove its potential as a marker of mortality since LDH values of patients who died after being admitted to the ICU before the $9^{\text {th }}$ day were already included in the unfavorable outcome group and not analyzed separately, our results suggest that future studies should investigate LDH as a marker of mortality.

The absence of significant differences for LDH changes on the $10^{\text {th }}$ and $11^{\text {th }}$ days of hospitalization may be due to the low number $(n=7)$ of determinations on these days in patients with unfavorable outcome. At this point in the hospital stay, many patients had already met the criteria for unfavorable outcome, so $\mathrm{LDH}$ values on these days were no longer useful as a predictive factor. Similarly, on the $9^{\text {th }}$ day of hospitalization, LDH was determined in only 10 patients with unfavorable outcome. Thus, it would be very interesting to carry out a prospective study specifically aimed at examining the prognostic value of the percentage of change in $\mathrm{LDH}$ from baseline values in the period comprising the $7^{\text {th }}$ through the $11^{\text {th }}$ days of hospitalization in COVID-19 patients.

Some studies found significant differences in ferritin values between patients with different outcomes $(3,19)$. However, these studies reported only absolute values and did not delve into the true prognostic capacity of the parameter. Although we found significant differences between the percentage change in ferritin on the $9^{\text {th }}$ day, the AUC in the ROC analysis was low, so there were no cutoffs that yielded good NPV and PPV. It is important to note that the mean percentage change in ferritin during the second week was positive even in patients with favorable outcomes. Although the increase was higher in patients with unfavorable outcomes, ferritin concentrations also continued to increase in many patients with good outcomes even after one week of hospitalization. Ferritin is an acutephase reactant, so sustained increases in ferritin over time even in cases with good outcomes are not surprising given the marked inflammatory 
character of COVID-19, including macrophage activation syndrome or cytokine storm $(33,34)$. A similar increase has also been reported in patients with good outcomes for another acute phase reactant, C-reactive protein (35).

We want to emphasize some limitations of our study. This was a retrospective done at a single center, limiting the number of patients and determinations that could be included. Thus, caution is warranted when extrapolating our results to other scenarios. Larger, prospective multicenter studies would be necessary to confirm our results. As our understanding of COVID-19 increases and more effective treatments become available, the cutoffs and predictive values of these parameters may change.

In conclusion, we found that increases in LDH from the baseline level (in the hospital admission day) above $15 \%$ on the 9 th day of hospitalization for COVID-19, predicts an unfavorable outcome with an $80 \%$ probability; the lack of an increase in LDH on the 9th day of hospitalization, rules out an unfavorable outcome with a $96 \%$ probability. In our study, ferritin was not useful for predicting the progression of COVID-19.
Abreviation
ICU: intensive care units
LDH: lactate dehydrogenase
ROC: receiver operating characteristic curve PPV: positive predictive value
AUC: area under the curve
NPV: negative predictive value

\section{Acknowledgments}

Not applicable.

\section{Author Contributions}

$\mathrm{AV}, \mathrm{GMC}$ and CCR conceived and designed the protocol. All authors performed data collection and analysis. AV and NE contributed to manu- script preparation. All authors read and approved the final manuscript.

\section{Research funding}

None declared.

\section{Competing interests}

The authors declare that there is no conflict of interests to disclose

\section{References}

1. World Health Organization, Coronavirus disease (COVID-19) pandemic. Available from: https://www. who.int/emergencies/diseases/novel-coronavirus-2019. Accessed June 1, 2020.

2. Cao W, Li T. COVID-19: towards understanding of pathogenesis. Cell Res. 2020; 30:367-69. DOI: 10.1038/s41422-020-0327-4

3. Bonetti G, Manelli F, Patroni A, Bettinardi A, Borrelli G, Fiordalisi G, et al. Laboratory predictors of death from coronavirus disease 2019 (COVID-19) in the area of Valcamonica, Italy. Clin Chem Lab Med. 2020; 58(7):1100-05. DOI: 10.1515/cclm-2020-0459

4. Wang K, Zuo P, Liu Y, Zhang M, Zhao X, Xie S, et al. Clinical and laboratory predictors of in-hospital mortality in patients with COVID-19: a cohort study in Wuhan, China. Clin Infect Dis. 2020;19;71(16):2079-88. DOI: $10.1093 / \mathrm{cid} / \mathrm{ciaa} 538$

5. Tian W, Jiang W, Yao Y, Nicholson CJ, Li RH, Sigurslide $\mathrm{HH}$, et al. Predictors of mortality in hospitalized COVID-19 patients: A systematic review and meta-analysis. J Med Virol. 2020;92(10):1875-83. DOI: 10.1002/jmv.26050

6. Zhou F, Yu T, Du R, Fan G, Liu Y, Liu Z, et al. Clinical course and risk factors for mortality of adult inpatients with COVID-19 in Wuhan, China: a retrospective cohort study. Lancet. 2020;395(10229):1054-62. DOI: 10.1016/S0140-6736(20)30566-3

7. $\mathrm{Li} \mathrm{Y,} \mathrm{Hu} \mathrm{Y,} \mathrm{Yu} \mathrm{J,} \mathrm{Ma} \mathrm{T.} \mathrm{Retrospective} \mathrm{analysis} \mathrm{of}$ laboratory testing in 54 patients with severe- or critical-type 2019 novel coronavirus pneumonia. Lab Invest. 2020;100(6):794-800. DOI: 10.1038/s41374-0200431-6

8. Henry BM, De Olivera MHS, Benoit S, Plebani M, 
Lippi G. Hematologic, biochemical and immune biomarker abnormalities associated with severe illness and mortality in coronavirus disease 2019 (COVID-19): a meta-analysis. Clin Chem Lab Med. 2020;58(7):102128. DOI: $10.1515 / \mathrm{cclm}-2020-0369$

9. Cecconi M, Piovani D, Brunetta E, Aghemo A, Greco M, Cicarelli M, et al. Early Predictors of Clinical Deterioration in a Cohort of 239 Patients Hospitalized for Covid-19 Infection in Lombardy, Italy. J Clin Med. 2020;9(5):e1548. DOI: 10.3390/jcm9051548

10. Health Ministry of Spain, Covid-19 situation in Spain. Available from: https://www.mscbs.gob.es/profesionales/saludPublica/ccayes/alertasActual/nCov-China/ situacionActual.htm. Accessed July 14, 2020.

11. Metropolitan Area of Barcelona, Metropolitan Barcelona information on COVID-19. Available from: http:// www.amb.cat/es/web/area-metropolitana/covid19. Accessed July 14, 2020.

12. Lippi G, Plebani M. Laboratory abnormalities in patients with COVID-2019 infection. Clin Chem Lab Med. 2020;58(7):1131-34. DOI: 10.1515/cclm-20200198

13. Sun Y, Dong Y, Wang L, Xie H, Li B, Chang C, et al. Characteristics and prognostic factors of disease severity in patients with COVID-19: The Beijing experience. J Autoimmun. 2020. DOI: 10.1016/j.jaut.2020.102473

14. Julián-Jimenez A, González del Castillo J, Candel F. Usefulness and prognostic value of biomarkers in patients with community-acquired pneumonia in the emergency department. Med Clin (Barc). 2017;148(11):50110. DOI: $10.1016 /$ j.medcle.2017.04.033

15. Li X, Wang L, Yan S, Xiang L, Zhu J, Shen B, et al. Clinical characteristics of 25 death cases with COVID-19: A retrospective review of medical records in a single medical center, Wuhan, China. Int J Infect Dis. 2020;94:128-32. DOI: 10.1016/j.ijid.2020.03.053

16. Liu J, Liu S. The management of coronavirus disease 2019 (COVID-19). J Med Virol. 2020; 92(10):2146-51. DOI: $10.1002 / j m v .25965$

17. Luo W, Lin Y, Yao X, Shi Y, Lu F, Wang Z, et al. Clinical findings of 35 cases with novel coronavirus pneumonia outside of Wuhan. Pulmonology. 2020. DOI: 10.21203/rs.3.rs-22554/v1

18. Guan WJ, Ni ZY, Hu Y. Clinical characteristics of coronavirus disease 2019 in China. N Engl J Med. 2020;382(18):1708-20. DOI: 10.1056/NEJMoa2002032
19. Liu T, Zhang J, Yang Y, Ma H, Li Z, Zhang J. The potential role of IL-6 in monitoring severe case of coronavirus disease 2019. EMBO Mol Med. 2020;12(7):e12421. DOI: $10.15252 / \mathrm{emmm} .202012421$

20. Wang D, Hu B, Hu C, Zhu F, Liu X, Zhang J, et al. Clinical characteristics of 138 hospitalized patients with 2019 novel coronavirus-infected pneumonia in Wuhan, China. JAMA. 2020;323(11):1061-69. DOI: 10.1001/ jama.2020.1585

21. Lian J, Jin X, Hao S, Cai H, Zhang S, Zheng L, et al. Clinical characteristics of 25 death cases with COVID-19: A retrospective review of medical records in a single medical center, Wuhan, China. Clin Infect Dis. 2020;94:128-32. DOI: 10.1016/j.ijid.2020.03.053

22. Huang C, Wang Y, Li X, Ren L, Zhao J, Hu Y, et al. Clinical features of patients infected with 2019 novel coronavirus in Wuhan, China. Lancet. 2020;395:497506. DOI: 10.1016/S0140-6736(20)30183-5

23. Weiss P. Clinical course and mortality risk of severe COVID-19. Lancet. 2020;395(10229):1014-15. DOI: 10.1016/S0140-6736(20)30633-4

24. Richardson S, Hirsch JS, Narasimhan M, Crawford J, McGinn T, Davidson KW, et al. Presenting Characteristics, Comorbidities, and Outcomes Among 5700 Patients Hospitalized with COVID-19 in the New York City Area. JAMA. 2020;323(20):2052-59. DOI: 10.1001/jama.2020.6775

25. Mo P, Xing Y, Xiao Y, Deng L, Zhao Q, Wang H, et al. Clinical characteristics of refractory COVID-19 pneumonia in Wuhan, China. Clin Infect Dis. 2020. DOI: 10.1093/cid/ciaa270

26. Liang W, Liang H, Ou L, Chen B, Chen A, Li C, et al. Development and Validation of a Clinical Risk Score to Predict the Occurrence of Critical Illness in Hospitalized Patients With COVID-19. JAMA. Intern Med 2020;1;180(8):1081-89. DOI: 10.1001/jamainternmed.2020.2033

27. Ji D, Zhang D, Xu J, Chen Z, Yang T, Zhao P, et al. Prediction for Progression Risk in Patients with COVID-19 Pneumonia: The CALL Score. Clin Infect Dis. 2020;12;71(6):1393-99. DOI: 10.1093/cid/ciaa414

28. Zhao Z, Chen A, Hou W, Graham JM, Li H, Richman PS, et al. Prediction model and risk scores of ICU admission and mortality in COVID-19. PLoS ONE. 2020;30;15(7):e0236618. DOI: 10.1371/journal. pone. 0236618 
29. Jean SS, Lee PI, Hsueh PRJ, et al. Treatment options for COVID-19: The reality and challenges. Microbiol Immunol Infect. 2020;53(3):436-43. DOI: 10.1016/j. jmii.2020.03.034

30. Grein J. Compassionate Use of Remdesivir for Patients with Severe Covid-19. N Engl J Med. 2020;382(24):2327-36. DOI: 10.1056/NEJMoa2007016

31. Liu YJ, Yang YL, Xu Y. What we learned from SARS may provide important insights into understanding and management of coronavirus disease 2019. Zhonghua Jie He He Hu Xi Za Zhi. 2020;339-44.

32. Kerboua KE. NLR: A Cost-effective Nomogram to Guide Therapeutic Interventions in
COVID-19. Immunol Invest. 2020;1:1-9. DOI: $10.1080 / 08820139.2020 .1773850$

33. McGonagle D, Sharif K, O'Regan A. The Role of Cytokines including Interleukin-6 in COVID-19 induced Pneumonia and Macrophage Activation Syndrome-Like Disease. Autoimmun Rev. 2020;19(6):102537. DOI: 10.1016/j.autrev.2020.102537

34. Moore JB and June CH. Cytokine release syndrome in severe COVID-19. Science. 2020;368(6490):473-74. DOI: $10.1126 /$ science.abb8925

35. Qin C, Zhou L, Hu Z, Zhang S, Yang Y, Tao Y, et al. Dysregulation of immune response in patients with COVID-19 in Wuhan, China. Clin Infect Dis. 2020;71(15):762-768. DOI: $10.1093 / \mathrm{cid} / \mathrm{ciaa} 248$ 